\title{
Tight bounds for the Pearle-Braunstein-Caves chained inequality without the fair-coincidence assumption
}

\author{
Jonathan Jogenfors* and Jan-Åke Larsson ${ }^{\dagger}$ \\ Institutionen för systemteknik, Linköpings Universitet, 58183 Linköping, Sweden
}

(Received 22 June 2017; published 1 August 2017)

\begin{abstract}
In any Bell test, loopholes can cause issues in the interpretation of the results, since an apparent violation of the inequality may not correspond to a violation of local realism. An important example is the coincidence-time loophole that arises when detector settings might influence the time when detection will occur. This effect can be observed in many experiments where measurement outcomes are to be compared between remote stations because the interpretation of an ostensible Bell violation strongly depends on the method used to decide coincidence. The coincidence-time loophole has previously been studied for the Clauser-Horne-Shimony-Holt and Clauser-Horne inequalities, but recent experiments have shown the need for a generalization. Here, we study the generalized "chained" inequality by Pearle, Braunstein, and Caves (PBC) with $N \geqslant 2$ settings per observer. This inequality has applications in, for instance, quantum key distribution where it has been used to reestablish security. In this paper we give the minimum coincidence probability for the PBC inequality for all $N \geqslant 2$ and show that this bound is tight for a violation free of the fair-coincidence assumption. Thus, if an experiment has a coincidence probability exceeding the critical value derived here, the coincidence-time loophole is eliminated.
\end{abstract}

DOI: 10.1103/PhysRevA.96.022102

\section{INTRODUCTION}

In recent years there has been an increased interest in the "chained" generalization by Pearle, Braunstein, and Caves (PBC) [1,2] of the CHSH [3,4] inequality due to its applications in reestablishing a full Bell violation. An important application is quantum key distribution (QKD) based on the Franson interferometer [5] where it is known [6-8] that the $\mathrm{CHSH}$ inequality is insufficient as a security test. If the switch to the full PBC is made, full security can be reestablished $[7,9]$.

Where the standard $\mathrm{CHSH}$ inequality is limited to two possible measurement settings per observer, the PBC inequality generalizes this to $N \geqslant 2$ settings. In order for Fransonbased systems to function, $N \geqslant 3$ is required at the cost of significantly higher experimental requirements. Specifically, such an experiment requires a very high visibility, and until recently it was believed [7] that these requirements were too impractical to achieve. Recent works [9], however, showed it possible to meet these requirements by reaching a full violation of the PBC inequality for $N=3,4$, and 5 with visibility in excess of $94.63 \%$.

Compared to other types of QKD such as BB84 [10] and E91 [11], the Franson design promises a simpler approach with fewer moving parts. This advantage could allow the Franson system to pave the way for commercial applications and widespread QKD adoption by reducing end-user complexity [8]. Therefore, the possibility of reestablishing full security in the Franson interferometer is a strong motivation of further study of the PBC inequality.

Previous works $[12,13]$ have shown that the $\mathrm{CHSH}$ and $\mathrm{CH}$ inequalities are vulnerable to the coincidence-time loophole which relates to the problem of attributing detector clicks to the correct pair of events. Bipartite Bell experiments measure correlations of outcomes between remote stations, and as this is done for each pair of detections, one must reliably

\footnotetext{
*jonathan.jogenfors@liu.se

†jan-ake.larsson@liu.se
}

decide which detector clicks correspond to which pair. This is more difficult than it might first appear due to high levels of nondetections, jitter in detection times, and dark counts. If coincidences are lost, one needs to apply the "fair-coincidence" assumption [13], i.e., that the outcome statistics is not skewed from these losses. According to [13], this fair-coincidence assumption appears to have been implicitly made in at least every experiment before 2015 .

This paper formally derives bounds for the coincidence probability so that a violation of the PBC inequality can be performed without the fair-coincidence assumption. Therefore, if the coincidence probability is high enough we can eliminate the coincidence-time loophole. It should be noted that switching to the generalized PBC inequality comes at a cost. As shown by [14], the minimum required detection efficiency is strictly increasing with $N$. Similarly, the PBC inequality in general has higher requirements for the coincidence probability than the $\mathrm{CHSH}$ inequality.

We begin by formally defining the coincidence probability for PBC-based experiments, followed by a sufficient condition for eliminating the coincidence-time loophole. Then, we show that our bound is tight by constructing a classical model that precisely reproduces the output statistics whenever the losses exceed the bound. Finally, we conclude that our results reduce to the special case of CHSH [12] by choosing $N=2$ and compare with the corresponding limits on detection efficiency [14].

\section{COINCIDENCE-TIME LOOPHOLE}

We use the symbol $\lambda$ for the hidden variable, which can take values in a sample space $\Lambda$, that in turn is the domain of random variables $A(\lambda)$ and $B(\lambda)$ denoting the measurement outcomes at Alice's and Bob's measurement stations, respectively. We further assume that the space $\Lambda$ has a probability measure $P$ which induces an expectation value $E$ in the standard way. We now give the formal definition of the PBC inequality $[1,2]$.

Theorem 1 (Pearle-Braunstein-Caves). Let $N$ be an integer $\geqslant 2$ and $i, j$, and $k$ be integers between 1 and $2 N$, and assume the following three prerequisites to hold almost everywhere. 
(i) Realism: measurement results can be described by probability theory, using two families of random variables $A_{i, j}, B_{i, j}$, e.g.,

$$
\begin{aligned}
A_{i, j}: \Lambda & \rightarrow V \\
\lambda & \mapsto A_{i, j}(\lambda), \\
B_{i, j}: \Lambda & \rightarrow V \\
\lambda & \mapsto B_{i, j}(\lambda) .
\end{aligned}
$$

(ii) Locality: a measurement result should be independent of the remote setting; e.g., for $k \neq i, l \neq j$ we have

$$
A_{i, j}(\lambda)=A_{i, l}(\lambda), \quad B_{i, j}(\lambda)=B_{k, j}(\lambda) .
$$

(iii) Measurement result restriction: the results may only range from -1 to +1 ,

$$
V=\{x \in \mathbb{R} ;-1 \leqslant x \leqslant+1\} .
$$

Then, by defining

$$
\begin{aligned}
S_{N} \stackrel{\text { def }}{=} & \left|E\left(A_{1} B_{1}\right)+E\left(A_{2} B_{1}\right)\right| \\
& +\left|E\left(A_{2} B_{2}\right)+E\left(A_{3} B_{2}\right)\right|+\cdots \\
& +\left|E\left(A_{N} B_{N}\right)-E\left(A_{1} B_{N}\right)\right|,
\end{aligned}
$$

we get

$$
S_{N} \leqslant 2 N-2 \text {. }
$$

The proof consists of simple algebraic manipulations, adding of integrals, and an application of the triangle inequality $[1,2]$.

The right-hand value of inequality (5) is the highest value $S_{N}$ can attain with a local realist model. Compare this with the prediction of quantum mechanics $[1,2]$ :

$$
S_{N}=2 N \cos \left(\frac{\pi}{2 N}\right) \text {. }
$$

Note that $2 N \cos (\pi / 2 N)>2 N-2$ which, in the spirit of Bell [4], shows that the outcomes of a quantum-mechanical experiment cannot be explained in local realist terms.

Computing the Bell value requires computing the correlation between outcomes at remote stations. Importantly, data must be gathered in pairs, so that products such as $A_{1} B_{1}$ can be computed [see Eq. (4)]. Experimentally, this is done by letting a source device generate pairs of (possibly entangled) particles that are sent to Alice and Bob for measurement. Detectors at either end record the measurement outcomes, and as previously mentioned there will always be variations on the detection times due to experimental effects. As a consequence, it is not always obvious which detector clicks correspond to which pairs of particles.

After a number of trials, Alice and Bob must determine in which trial if they have coincidence (simultaneous clicks at Alice and Bob), a single event (only one party gets a detection), or no detection at all. This is especially pronounced if the experimental setup uses down-conversion where a continuouswave laser pumps a nonlinear crystal in order to spontaneously create pairs of entangled photons. In that case the emission time is uniformly distributed over the duration of the experiment so it becomes a probabilistic process that further complicates pair detection.
A typical strategy used in quantum optics experiments to reduce the influence of noise in a Bell experiment is to have a time window of size $\Delta T$ around, for example, Alice's detection event $[15,16]$. If a detection event has occurred at Bob's side within this window it is counted as a coincident pair. This is a nonlocal strategy as it involves comparing data between remote stations and is used in many experiments.

For the experimenter it is tempting to choose a small $\Delta T$ since it filters out noise and therefore increases the measured Bell value. At this point, there is apparently no immediately obvious drawback of picking a very small $\Delta T$. However, rejecting experimental data in a Bell experiment modifies the underlying statistical ensemble and it is known [17] to lead to inflated Bell values and a false violation of the Bell inequality. This is a so-called loophole that can arise in Bell testing, and many such loopholes have been studied in recent years (see [18] for a review).

A coincidence window that is too small discards some truly coincident events as noise. Therefore, the Bell value measurement only occurs on a subset of the statistical ensemble which means a number of events are not accounted for when the Bell value is computed. While the Bell value $S_{N}$ from Theorem 1 is in violation of a Bell inequality, this violation might be a mirage. Specifically, the loophole that arises from choosing a small $\Delta T$ is called the coincidence-time loophole and has previously been studied [12] for the special CHSH case $N=2$. In addition, more recent works [13] derive similar bounds for the $\mathrm{CH}$ [19] inequality.

We generalize the results previously obtained for the special case of $\mathrm{CHSH}$ by deriving tight bounds for the coincidencetime loophole in the PBC inequality (Theorem 1 ) for all $N \geqslant 2$. This contribution will be useful for future experiments investigating, among others, Franson-based QKD. Other works [14] have studied the effects of reduced detector efficiency for the full PBC inequality, which in turn is a generalization of an older result [17] that only discussed the special $\mathrm{CHSH}$ case.

For the rest of this paper, Alice and Bob perform measurements on some underlying, possibly quantum, system. Their measurements are chosen from $\left\{A_{i}\right\}$ and $\left\{B_{j}\right\}$, respectively, i.e., sets of $N$ measurement settings each. As discussed by Larsson and Gill [12], Alice's and Bob's choice of measurement settings might influence whether an event is coincident or not. Following the formalism in [17] we will therefore model noncoincident settings $\lambda$ as subsets of $\Lambda$ where the random variables $A_{i}(\lambda)$ and $B_{i}(\lambda)$ are undefined. We must therefore modify the expectation values in Eq. (4) to be conditioned on coincidence in order for $S_{N}$ to be well defined [see Eq. (10)]. The time of arrival at Alice's and Bob's measurement stations is defined as

$$
\begin{aligned}
T_{i, j}: \Lambda & \rightarrow \mathbb{R} \\
\lambda & \mapsto T_{i, j}(\lambda), \\
T_{i, j}^{\prime}: \Lambda & \rightarrow \mathbb{R} \\
\lambda & \mapsto T_{i, j}^{\prime}(\lambda),
\end{aligned}
$$

respectively. Since this notation will become cumbersome, we will introduce a simplification. Let $\quad\left\{b_{i}\right\}_{1}{ }^{2 N}=\{1,1,2,2, \ldots, N, N\} \quad$ and $a_{i}$ rotated one step so that $\left\{a_{i}\right\}_{1}{ }^{2 N}=\{1,2,2, \ldots, N, N, 1\}$. Then 
$\left\{\left(a_{i}, b_{i}\right)\right\}_{1}{ }^{2 N}=\{(1,1),(2,1),(2,2),(3,2), \ldots,(N, N),(1, N)\}$.

This allows us to define subsets of $\Lambda$ as the sets on which Alice's and Bob's measurement settings give coincident outcomes. For $1 \leqslant i \leqslant 2 N$ we have

$$
\Lambda_{i} \stackrel{\text { def }}{=}\left\{\lambda:\left|T_{a_{i}, b_{i}}(\lambda)-T_{a_{i}, b_{i}}^{\prime}(\lambda)\right|<\Delta T\right\} .
$$

We can now calculate the probability of coincidence as

$$
\gamma_{N} \stackrel{\text { def }}{=} \inf _{i} P\left(\Lambda_{i}\right)
$$

Finally, for $1 \leqslant i \leqslant 2 N$ we have the conditional expectation defined as

$$
E\left(X_{i} \mid \Lambda_{i}\right) \stackrel{\text { def }}{=} \int_{\Lambda_{i}} X_{i}(\lambda) d P(\lambda)
$$

where we use the convenient shorthand

$$
X_{i} \stackrel{\text { def }}{=} A_{a_{i}} B_{b_{i}}
$$

for the product of the outcomes of Alice and Bob.

\section{PBC INEQUALITY WITH COINCIDENCE PROBABILITY}

We can now restate Theorem 1 in terms of coincidence probability.

Theorem 2 (PBC with coincidence probability). Let $N$ be an integer $\geqslant 2$ and $i, j$, and $k$ be integers between 1 and $2 N$, and assume the prerequisites $(i)-($ ii i ) of Theorem 1 hold almost everywhere together with the following.

(iv) Coincident events: correlations are obtained on $\Lambda_{i} \subset \Lambda$.

Then by defining

$$
\begin{aligned}
S_{C, N} \stackrel{\text { def }}{=} & \left|E\left(X_{1} \mid \Lambda_{1}\right)+E\left(X_{2} \mid \Lambda_{2}\right)\right|+\cdots \\
& +\left|E\left(X_{2 N-1} \mid \Lambda_{2 N-1}\right)-E\left(X_{2 N} \mid \Lambda_{2 N}\right)\right|
\end{aligned}
$$

we get

$$
S_{C, N} \leqslant \frac{4 N-2}{\gamma_{N}}-2 N
$$

The remainder of this section is dedicated to proving this result. Note that while the proof of Theorem 1 consists of adding expectation values, this cannot be done for Theorem 2 since $\Lambda_{i} \neq \Lambda_{j}$ in general. Again, the ensemble changes with Alice's and Bob's measurement settings, so the ensemble that Theorem 1 implicitly acts upon is really

$$
\Lambda_{I} \stackrel{\text { def }}{=} \bigcap_{i=1}^{2 N} \Lambda_{j}
$$

i.e., the intersection of all coincident subspaces of $\Lambda$. In other words, prerequisites $(i)-($ iii $)$ yield

$$
\begin{aligned}
& \left|E\left(A_{1} B_{1} \mid \Lambda_{I}\right)+E\left(A_{2} B_{1} \mid \Lambda_{I}\right)\right|+\cdots \\
& +\left|E\left(A_{N} B_{N} \mid \Lambda_{I}\right)-E\left(A_{1} B_{N} \mid \Lambda_{I}\right)\right| \leqslant 2 N-2,
\end{aligned}
$$

which again is a more precise restatement of Theorem 1 where we stress the conditional part. An experiment, however, will give us results on the form $E\left(X_{i} \mid \Lambda_{i}\right)$, i.e., $E\left(X_{i} \mid \Lambda_{I}\right)$ is unavailable to the experimenter. We therefore need to bridge the gap between experimental data and Theorem 2, so we define the following quantity which will act as a stepping stone:

$$
\delta \stackrel{\text { def }}{=} \inf _{i} \frac{P\left(\bigcap_{j=1}^{2 N} \Lambda_{j}\right)}{P\left(\Lambda_{i}\right)}=\inf _{i} P\left(\bigcap_{j \neq i} \Lambda_{j} \mid \Lambda_{i}\right) .
$$

Note that it is possible for the ensemble $\Lambda_{I}$ to be empty, but only when $\delta=0$ and then inequality (13) is trivial. We can therefore assume $\delta>0$ for the rest of the proof and our goal now is to give a lower bound to $\delta$ in terms of the coincidence probability $\gamma_{N}$. We fix $i$ and apply Boole's inequality,

$$
P\left(\bigcap_{j \neq i} \Lambda_{j} \mid \Lambda_{i}\right) \geqslant 2 N-2+\sum_{j \neq i} P\left(\Lambda_{j} \mid \Lambda_{i}\right),
$$

and rewrite the summation terms:

$$
\begin{aligned}
P\left(\Lambda_{j} \mid \Lambda_{i}\right)= & \frac{P\left(\Lambda_{i} \cap \Lambda_{j}\right)}{P\left(\Lambda_{i}\right)} \\
= & \frac{P\left(\Lambda_{i}\right)+P\left(\Lambda_{j}\right)-P\left(\Lambda_{i} \cup \Lambda_{j}\right)}{P\left(\Lambda_{i}\right)} \\
& \geqslant 1+\frac{P\left(\Lambda_{j}\right)-1}{P\left(\Lambda_{i}\right)} \geqslant 1+\frac{\gamma_{N}-1}{\gamma_{N}} \\
= & 2-\frac{1}{\gamma_{N}} .
\end{aligned}
$$

Inserting inequality (18) into inequality (17) we get

$$
\begin{gathered}
P\left(\bigcap_{j \neq i} \Lambda_{j} \mid \Lambda_{i}\right) \geqslant 2 N-2+(2 N-1) \\
\left(2-\frac{1}{\gamma_{N}}\right)=2 N-\frac{2 N-1}{\gamma_{N}},
\end{gathered}
$$

and as inequality (18) is independent of $i$, inserting into Eq. (16) gives

$$
\delta \geqslant 2 N-\frac{2 N-1}{\gamma_{N}},
$$

and this is the desired lower bound. We now bound $S_{C, N}$ from above by adding and subtracting $\delta E\left(X_{i} \mid \Lambda_{I}\right)$ in every term before applying the triangle inequality and use Eq. (15):

$$
\begin{aligned}
S_{C, N}= & \mid E\left(X_{1} \mid \Lambda_{1}\right)-\delta E\left(X_{1} \mid \Lambda_{I}\right)+\delta E\left(X_{1} \mid \Lambda_{I}\right)+E\left(X_{2} \mid \Lambda_{2}\right) \\
& -\delta E\left(X_{2} \mid \Lambda_{I}\right)+\delta E\left(X_{2} \mid \Lambda_{I}\right) \mid+\cdots \\
& +\mid E\left(X_{2 N-1} \mid \Lambda_{2 N-1}\right)-\delta E\left(X_{2 N-1} \mid \Lambda_{I}\right) \\
& +\delta E\left(X_{2 N-1} \mid \Lambda_{I}\right)-E\left(X_{2 N} \mid \Lambda_{2 N}\right) \\
& +\delta E\left(X_{2 N} \mid \Lambda_{I}\right)-\delta E\left(X_{2 N} \mid \Lambda_{I}\right) \mid \\
\leqslant & \delta\left(\left|E\left(X_{1} \mid \Lambda_{I}\right)+E\left(X_{2} \mid \Lambda_{I}\right)\right|+\cdots\right. \\
& \left.+\left|E\left(X_{2 N-1} \mid \Lambda_{I}\right)-E\left(X_{2 N} \mid \Lambda_{I}\right)\right|\right) \\
& +\sum_{i=1}^{2 N}\left|E\left(X_{i} \mid \Lambda_{i}\right)-\delta E\left(X_{i} \mid \Lambda_{I}\right)\right| \\
\leqslant & \delta S_{N}+\sum_{i=1}^{2 N}\left|E\left(X_{i} \mid \Lambda_{i}\right)-\delta E\left(X_{i} \mid \Lambda_{I}\right)\right| .
\end{aligned}
$$


To give an upper bound to the last sum, we need the following lemma.

Lemma 1. For $1 \leqslant i \leqslant 2 N$ and $0 \leqslant \delta \leqslant 1$ we have the following inequality:

$$
\left|E\left(X_{i} \mid \Lambda_{i}\right)-\delta E\left(X_{i} \mid \Lambda_{I}\right)\right| \leqslant 1-\delta .
$$

Proof 1. It is clear that $\Lambda_{I} \subset \Lambda_{i}$. We can therefore split $\Lambda_{i}$ in two disjoint sets: $\Lambda_{*} \stackrel{\text { def }}{=} \Lambda_{i} \backslash \Lambda_{I}$ and $\Lambda_{I}$. It follows that $\Lambda_{I} \cup \Lambda_{*}=\Lambda_{i}$ and we have

$$
\begin{aligned}
& \left|E\left(X_{i} \mid \Lambda_{i}\right)-\delta E\left(X_{i} \mid \Lambda_{I}\right)\right| \leqslant\left|P\left(\Lambda_{*} \mid \Lambda_{i}\right) E\left(X_{i} \mid \Lambda_{*}\right)\right| \\
& \quad+\left|P\left(\Lambda_{I} \mid \Lambda_{i}\right) E\left(X_{i} \mid \Lambda_{I}\right)-\delta E\left(X_{i} \mid \Lambda_{I}\right)\right| \\
& \leqslant P\left(\Lambda_{*} \mid \Lambda_{i}\right) E\left(\left|X_{i}\right| \mid \Lambda_{*}\right)+\left(P\left(\Lambda_{I} \mid \Lambda_{i}\right)-\delta\right) E\left(\left|X_{i}\right| \mid \Lambda_{I}\right) \\
& \leqslant P\left(\Lambda_{*} \mid \Lambda_{i}\right)+P\left(\Lambda_{I} \mid \Lambda_{i}\right)-\delta=1-\delta .
\end{aligned}
$$

Lemma 1 gives us

$$
\sum_{i=1}^{2 N}\left|E\left(X_{i} \mid \Lambda_{i}\right)-\delta E\left(X_{i} \mid \Lambda_{I}\right)\right| \leqslant 2 N(1-\delta) .
$$

The final step is to use inequalities (5), (20), and (24) on inequality (21), which proves the desired result.

\section{MINIMUM COINCIDENCE PROBABILITY}

The right-hand side of inequality (13) increases as $\gamma_{N}$ goes down so there exists a unique $\gamma_{N}$ so the bound on $S_{C, N}$ coincides with the quantum-mechanical prediction in Eq. (6). We define this critical coincidence probability as $\gamma_{\mathrm{crit}, N}$ and find it by solving the following equation:

$$
2 N \cos \left(\frac{\pi}{2 N}\right)=\frac{4 N-2}{\gamma_{\text {crit }, N}}-2 N
$$

and get

$$
\gamma_{\text {crit }, N}=\frac{2 N-1}{2 N}\left(1+\tan ^{2}\left(\frac{\pi}{4 N}\right)\right) .
$$

What remains to show is that for all $\gamma_{N} \leqslant \gamma_{\text {crit, } N}$ there exists a local hidden variable (LHV) model that produces a $S_{C, N}$ that mimics the predictions of quantum theory. Formally, we have the following theorem.

Theorem 3. Let $N$ be an integer $\geqslant 2$. For every $\gamma_{N} \leqslant \gamma_{\text {crit }, N}$ it is possible to construct an LHV model fulfilling the prerequisites (i)-(iv) of inequality (13) so that

$$
S_{C, N}=2 N \cos \left(\frac{\pi}{2 N}\right) .
$$

We explicitly prove Theorem 3 by constructing the LHV model depicted in Fig. 1. Here, the hidden variable is on the form $(r, \theta)$ and uniformly distributed over $0 \leqslant r \leqslant 1$ and $0 \leqslant$ $\theta \leqslant 2 \pi$. The LHV model defines the random variables $A_{i}$ and $B_{i}$ and arrival times $T_{i}$ and $T_{i}^{\prime}$, where we adapt the shorthand from Eq. (11) to the definition in Eq. (7). We choose $\phi$ to be a function of $i$ in the following way for Alice's detector:

$$
\phi(i) \stackrel{\text { def }}{=} a_{i} \frac{\pi}{2 N}
$$

and the following way for Bob's detector:

$$
\phi(i) \stackrel{\text { def }}{=} b_{i} \frac{\pi}{2 N} .
$$

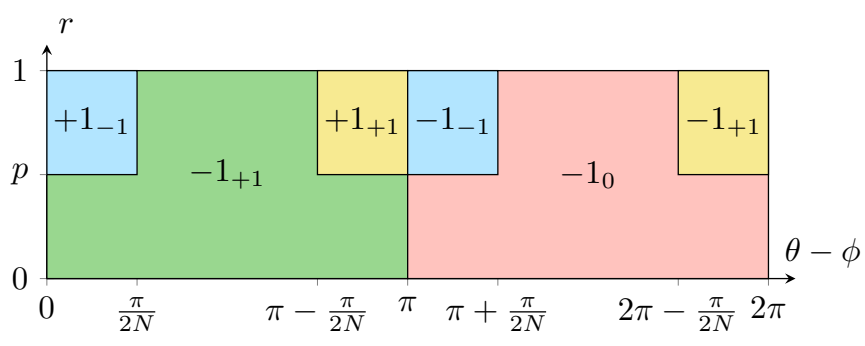

FIG. 1. LHV model that gives the outcomes for Alice's and Bob's detectors.

In Fig. 1, $\phi$ acts as a shift in the $\theta$ direction (with wraparound when neccessary). The case $i=1$ is depicted in Fig. 2, and by choosing $\Delta T=3 / 2$ we get coincidence for a time difference of 0 and 1 units (solid background), and noncoincidence for a time difference of two units (cross-hatched background). We compute the probability of coincidence in Fig. $2, P\left(\Lambda_{i}\right)=$ $(2 N-1+p) / 2 N$ and find that it is independent of $i$. Therefore,

$$
\gamma_{N}=(2 N-1+p) / 2 N
$$

In addition, for $1 \leqslant i \leqslant 2 N-1$,

$$
\begin{aligned}
P\left(X_{i}=+1 \mid \Lambda_{i}\right) & =\frac{P\left(X_{i}=+1\right)}{P\left(\Lambda_{i}\right)} \\
& =\frac{2 N-1}{2 N-1+p}
\end{aligned}
$$

and

$$
\begin{aligned}
P\left(X_{i}=-1 \mid \Lambda_{i}\right) & =\frac{P\left(X_{i}=+1 \cap \Lambda_{i}\right)}{P\left(\Lambda_{i}\right)} \\
& =\frac{p}{2 N-1+p},
\end{aligned}
$$

which gives

$$
\begin{aligned}
E\left(X_{i} \mid \Lambda_{i}\right) & =P\left(X_{i}=+1 \mid \Lambda_{i}\right)-P\left(X_{i}=-1 \mid \Lambda_{i}\right) \\
& =\frac{2 N-1-p}{2 N-1+p}
\end{aligned}
$$

for $1 \leqslant i \leqslant 2 N-1$. A similar calculation yields

$$
E\left(X_{2 N} \mid \Lambda_{2 N}\right)=-\frac{2 N-1-p}{2 N-1+p} .
$$

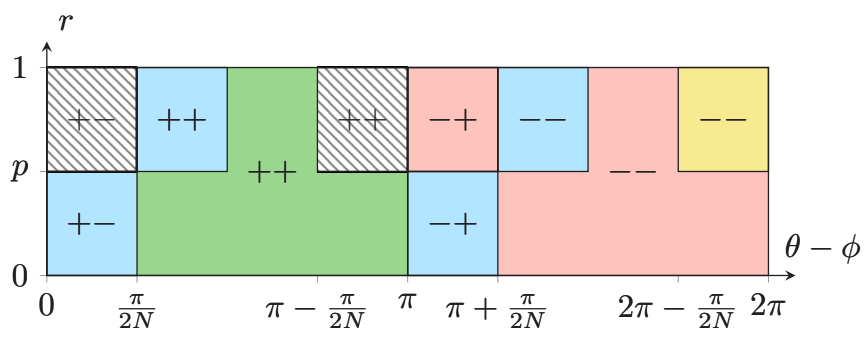

FIG. 2. Alice's and Bob's outcome patterns for the case $i=1$. The two plus and/or minus signs show Alice's and Bob's outcome, respectively. The cross-hatch areas show outcomes that are noncoincident given $\Delta T=3 / 2$. 
TABLE I. Critical coincidence probabilities $\gamma_{\text {crit }, N}$ and detection probabilities $\eta_{\text {crit }, N}$ for a loophole-free violation of the PBC equality for 2, 3, 4, and 5 measurement settings. Note that $N=2$ corresponds to the special $\mathrm{CHSH}$ case.

\begin{tabular}{lcc}
\hline \hline$N$ & $\gamma_{\text {crit }, N}$ & $\eta_{\text {crit }, N}$ \\
\hline 2 (CHSH) & $87.87 \%$ & $82.84 \%$ \\
3 & $89.32 \%$ & $86.99 \%$ \\
4 & $90.96 \%$ & $89.61 \%$ \\
5 & $92.26 \%$ & $91.37 \%$ \\
$N$ & \multicolumn{2}{c}{ Increases with $N$} \\
\hline \hline
\end{tabular}

We now insert the predictions of the LHV model into Eq. (12) to get

$$
\begin{aligned}
S_{\mathrm{LHV}, N} \stackrel{\text { def }}{=} & \left|E\left(X_{1} \mid \Lambda_{1}\right)+E\left(X_{2} \mid \Lambda_{2}\right)\right|+\cdots \\
& +\left|E\left(X_{2 N-1} \mid \Lambda_{2 N-1}\right)-E\left(X_{2 N} \mid \Lambda_{2 N}\right)\right| .
\end{aligned}
$$

As we want the LHV model to mimic the predictions of quantum mechanics [from Eq. (6)] we put

$$
S_{\mathrm{LHV}, N}=2 N \cos \left(\frac{\pi}{2 N}\right),
$$

which gives

$$
\frac{2 N-1-p}{2 N-1+p}=\cos \left(\frac{\pi}{2 N}\right) .
$$

Solving for $p$ we get

$$
p=(2 N-1) \tan ^{2}\left(\frac{\pi}{4 N}\right)
$$

and Eq. (30) then gives us

$$
\gamma_{N}=\frac{2 N-1}{2 N}\left(1+\tan ^{2}\left(\frac{\pi}{4 N}\right)\right)
$$

which coincides with $\gamma_{\text {crit }, N}$. The model in Fig. 1 is a constructive proof of Theorem 3 as it produces the same output statistics as quantum mechanics with coincidence probability $\gamma_{\text {crit }, N}$. We finally note that it is trivial to modify the LHV model to give any $\gamma \leqslant \gamma_{\text {crit }, N}$, which finishes the proof.

The LHV model in Fig. 1 mimics almost every statistical property of a truly quantum-mechanical experiment (see [12]) and shows it is possible to fake a violation of the $\mathrm{PBC}$ inequality if the coincidence probability is lower than the critical value. It is therefore important that any experiment relying on a PBC inequality violation takes the coincidence probability into account before ruling out a classical model.

As the number of measurement settings $N$ goes to infinity the critical coincidence probability $\gamma_{\text {crit }, N}$ goes to 1 . Therefore, achieving the required coincidence becomes harder as more measurement settings are used. If we define $\eta_{\text {crit, } N}$ as the minimum required detection efficiency for a violation of the PBC inequality free of the detection loophole (see [14] for full details) we get

$$
\eta_{\text {crit }, N}=\frac{2}{\frac{N}{N-1} \cos \left(\frac{\pi}{2 N}\right)+1}
$$

and note that $\gamma_{\text {crit }, N}>\eta_{\text {crit }, N}$ for all $N \geqslant 2$. In addition, the critical coincidence probability for the special CHSH case $N=2$ is $87.87 \%$, which agrees with previous works [12]. See Table I for critical probabilities for the cases $N=2,3,4,5$ and note that both $\gamma_{\mathrm{crit}, N}$ and $\eta_{\mathrm{crit}, N}$ are strictly increasing in $N$. Note that a loophole-free experiment requires both the coincidence probability and detection efficiency be in excess of their respective thresholds.

While reaching $\gamma_{\text {crit }, N}$ is less challenging for small $N$, some applications do require a PBC inequality with a higher number of settings. An example is the Franson interferometer [5], where postselection leads to a loophole for $N=2$ but not for $N \geqslant 3$ [7]. In fact, $N=5$ is optimal for that setup in terms of violation; however, Table I shows that the corresponding minimal coincidence probability is as high as $92.26 \%$, which is a considerable challenge.

\section{CONCLUSION}

The PBC inequality is a powerful tool for testing local realism in applications where the $\mathrm{CHSH}$ test is insufficient. We have found the minimum required coincidence probability for a violation of the $\mathrm{PBC}$ inequality without the fair-coincidence assumption. This bound is tight, so any application of the PBC inequality that relies on a violation of local realism must have at least this coincidence probability, unless the perilous fair-coincidence assumption is to be made. If not, and if the coincidence probability is below the critical threshold, an attacker can construct a local realist model from which all measurements can be predicted.
[1] P. Pearle, Hidden-variable example based upon data rejection, Phys. Rev. D 2, 1418 (1970).

[2] S. L. Braunstein and C. Caves, Wringing out better Bell inequalities, Ann. Phys. (N.Y.) 202, 22 (1990).

[3] J. F. Clauser, M. A. Horne, A. Shimony, and R. A. Holt, Proposed Experiment to Test Local Hidden-Variable Theories, Phys. Rev. Lett. 23, 880 (1969).

[4] J. S. Bell, On the Einstein-Podolsky-Rosen paradox, Physics (Long Island City, N. Y.) 1, 195 (1964).

[5] J. D. Franson, Bell Inequality for Position and Time, Phys. Rev. Lett. 62, 2205 (1989).
[6] S. Aerts, P. Kwiat, J.-Å. Larsson, and M. Zukowski, Two-Photon Franson-Type Experiments and Local Realism, Phys. Rev. Lett. 83, 2872 (1999).

[7] J. Jogenfors and J.-Å. Larsson, Energy-time entanglement, elements of reality, and local realism, J. Phys. A: Math. Theor. 47, 424032 (2014).

[8] J. Jogenfors, A. M. Elhassan, J. Ahrens, M. Bourennane, and J.-A. Larsson, Hacking the bell test using classical light in energy-time entanglement-based quantum key distribution, Sci. Adv. 1, e1500793 (2015). 
[9] M. Tomasin, E. Mantoan, J. Jogenfors, G. Vallone, J.-Å. Larsson, and P. Villoresi, High-visibility time-bin entanglement for testing chained Bell inequalities, Phys. Rev. A 95, 032107 (2017).

[10] C. H. Bennett and G. Brassard, Quantum cryptography: Public key distribution and coin tossing, in Proceedings of the IEEE International Conference on Computers, Systems, and Signal Processing (IEEE, New York, 1984), pp. 175-179.

[11] A. K. Ekert, Quantum Cryptography Based on Bell's Theorem, Phys. Rev. Lett. 67, 661 (1991).

[12] J.- A. Larsson and R. D. Gill, Bell's inequality and the coincidence-time loophole, Europhys. Lett. 67, 707 (2004).

[13] J.-Å. Larsson, M. Giustina, J. Kofler, B. Wittmann, R. Ursin, and S. Ramelow, Bell-inequality violation with entangled photons, free of the coincidence-time loophole, Phys. Rev. A 90, 032107 (2014).

[14] A. Cabello, J.-Å. Larsson, and D. Rodriguez, Minimum detection efficiency required for a loophole-free violation of the
Braunstein-Caves chained Bell inequalities, Phys. Rev. A 79, 062109 (2009).

[15] M. Giustina, A. Mech, S. Ramelow, B. Wittmann, J. Kofler, J. Beyer, A. Lita, B. Calkins, T. Gerrits, S. W. Nam, R. Ursin, and A. Zeilinger, Bell violation using entangled photons without the fair-sampling assumption, Nature (London) 497, 227 (2013).

[16] B. G. Christensen, A. Hill, P. G. Kwiat, E. Knill, S. W. Nam, K. Coakley, S. Glancy, L. K. Shalm, and Y. Zhang, Analysis of coincidence-time loopholes in experimental Bell tests, Phys. Rev. A 92, 032130 (2015).

[17] J.-A. Larsson, Bell's inequality and detector inefficiency, Phys. Rev. A 57, 3304 (1998).

[18] J.-A. Larsson, Loopholes in Bell inequality tests of local realism, J. Phys. A 47, 424003 (2014).

[19] J. F. Clauser and M. A. Horne, Experimental consequences of objective local theories, Phys. Rev. D 10, 526 (1974). 